\title{
Wild-type bone marrow transplant partially reverses neuroinflammation in progranulin-deficient mice
}

\author{
Yue Yang, Macarena S Aloi, Eiron Cudaback, Samuel R Josephsen, Samantha J Rice, Nikolas L Jorstad, C Dirk Keene \\ and Thomas J Montine
}

Frontotemporal dementia (FTD) is a neurodegenerative disease with devastating changes in behavioral performance and social function. Mutations in the progranulin gene (GRN) are one of the most common causes of inherited FTD due to reduced progranulin expression or activity, including in brain where it is expressed primarily by neurons and microglia. Thus, efforts aimed at enhancing progranulin levels might be a promising therapeutic strategy. Bone marrow (BM)derived cells are able to engraft in the brain and adopt a microglial phenotype under myeloablative irradiation conditioning. This ability makes BM-derived cells a potential cellular vehicle for transferring therapeutic molecules to the central nervous system. Here, we utilized BM cells from $\mathrm{Grn}^{+/+}$(wild type or wt) mice labeled with green fluorescence protein for delivery of progranulin to progranulin-deficient $\left(\mathrm{Grn}^{-/-}\right)$mice. Our results showed that wt bone marrow transplantation (BMT) partially reconstituted progranulin in the periphery and in cerebral cortex of $\mathrm{Grn}^{-1-}$ mice. We demonstrated a pro-inflammatory effect in vivo and in ex vivo preparations of cerebral cortex of $\mathrm{Grn}^{-1-}$ mice that was partially to fully reversed 5 months after BMT. Our findings suggest that BMT can be administered as a stem cell-based approach to prevent or to treat neurodegenerative diseases.

Laboratory Investigation (2014) 94, 1224-1236; doi:10.1038/labinvest.2014.113; published online 8 September 2014

Homozygous mutations in the progranulin gene (GRN) cause a disease of childhood and young adults called neuronal ceroid lipofuscinosis-11 (OMIM Entry: \#614706). ${ }^{1,2}$ GRN haploinsufficiency is a common cause of a form of dementia in middle-aged adults called frontotemporal dementia (FTD) that derives from a neurodegenerative disease called frontotemporal lobar degeneration (FTLD) with transactive response DNA-binding protein $43 \mathrm{kDa}$ (TDP43)-positive inclusions, or FTLD-TDP. ${ }^{2-11}$ Indeed, at least 70 different GRN mutations (http://www.molgen.ua.ac.be/ FTDMutations/), all of which result in progranulin deficiency, cause FTLD-TDP. ${ }^{12-14}$

Progranulin is expressed in diverse tissue including bone marrow (BM) and circulating immune cells as well as solid organs including the brain, and is present in plasma and cerebrospinal fluid. ${ }^{15-19}$ In brain, progranulin is expressed largely by neurons and microglia ${ }^{2,16}$ with increased expression in activated microglia. ${ }^{13,20}$ Progranulin is pleiotropic and is secreted into the extracellular fluid where it exerts autocrine and paracrine effects. ${ }^{21}$ Its direct actions include regulation of innate immunity ${ }^{22,23}$ and neurotrophism. ${ }^{24-28}$ Progranulin also is cleaved by multiple enzymes including neutrophil elastase, ${ }^{29}$ various matrix metalloproteinases, ${ }^{30}$ and proteinase $3^{31}$ to produce granulin that contributes to regulation of the cell cycle and inflammation, among other actions. ${ }^{32,33}$ Moreover, there is a complex interplay between progranulin and granulin cleavage products. For instance, full-length progranulin is anti-inflammatory while proteolytically released granulins can have the opposite action. ${ }^{22,29,34,35}$

Insight into the mechanisms of progranulin deficiency has been aided by development of mice homozygous deficient for progranulin $\left(\mathrm{Grn}^{-1-}\right),{ }^{36-38}$ which display increased innate immune activation, behavioral abnormalities, and neuropathologic changes that share some of the features of FTLDTDP. ${ }^{2,22,23}$ Mice hemizygous for Grn exhibit much milder pathologic and behavioral changes. ${ }^{2,39}$ Attempts to effectively reverse the deficits caused by progranulin deficiency have been attempted in cell culture using small molecules. ${ }^{40}$ We tested the hypothesis that bone marrow transplantation (BMT) from $\mathrm{Grn}^{+/+}$(wt) mice into $\mathrm{Grn}^{-1-}$ mice would result in increased levels of circulating and central nervous system (CNS) progranulin, and mitigate the pro-inflammatory changes in the CNS that characterize progranulin deficiency in younger adult mice.

Department of Pathology, University of Washington, Seattle, WA, USA

Correspondence: Dr Y Yang, PhD, Department of Pathology, Harborview Medical Center, University of Washington, 300 9th Avenue, Seattle, WA 98104, USA.

E-mail: yangyue@u.washington.edu

Received 13 June 2014; revised 9 July 2014; accepted 11 July 2014 


\section{MATERIAL AND METHODS Animals}

All mice were maintained in a C57BL/6 background and were congenic with identical MHC. ${ }^{41}$ BM-recipient mice were 2-month-old progranulin knock-out $\left(\mathrm{Grn}^{-1-}\right)$ or $\mathrm{Grn}^{+/+}$ (wt) mice and their non-transplanted littermates were used as controls. $\mathrm{Grn}^{-1-}$ mice on a C57BL/6 background ${ }^{22,37}$ were purchased from the Jackson Laboratory (B6.CgGrntm1.1Aidi/J catalog \#013175; Bar Harbor, ME). BM-donor mice were male wt mice homozygous for green fluorescent protein (GFP). GFP expression is under control of the $\beta$-actin promoter and cytomegalovirus enhancer. Mice were housed in standard laboratory conditions with a strict 12-h light/ dark cycle and with free access to mouse chow and water. All protocols were approved by the University of Washington Institutional Animal Care and Use Committee.

\section{Bone Marrow Transplantation}

BMT was performed in recipient wt or $\mathrm{Grn}^{-1-}$ mice using wt-GFP mice as donors. Recipient wt or $\mathrm{Grn}^{-1-}$ mice at 2 months of age received total body irradiation $(10.5 \mathrm{~Gy})$ in a single dose at $\sim 2$ Gy per minute from a Cesium-137 source (JL Shepherd, Model 81-14, San Fernando, CA). Previous studies ${ }^{42,43}$ have reported a $100 \%$ incidence of death at this dose. BM was isolated from 6-week-old male wt-GFP mice by flushing the femurs and tibias with RPMI media with $10 \%$ fetal bovine serum. The samples were combined, passed through a 25 -gauge needle filtered through a $70-\mu \mathrm{m}$ nylon mesh, and centrifuged. Erythrocytes were lysed in ammonium chloride potassium (ACK) buffer (Invitrogen, Carlsbad, CA) and the remaining leukocytes were resuspended in sterile PBS at a concentration of $\sim 5 \times 10^{6}$ viable nucleated cells per $200 \mu$ l. Irradiated wt or $\mathrm{Grn}^{-1-}$ mice received wt-GFP BM cells via retroorbital venous plexus injections 1 day after total body irradiation and were housed in autoclaved cages. Chimeric mice were then euthanized for analysis 5 months after transplantation.

\section{Tissue Collection and Processing}

Animals were anesthetized with 2.5\% tribromoethanol (Avertin; Sigma-Aldrich, St Louis, MO) 5 months post transplantation. Blood was drawn via cardiac puncture and processed for flow cytometry before the mice were transcardially perfused with ice-cold PBS. Brains were rapidly removed from the skulls and divided by mid-sagittal section. One hemibrain was dissected into anatomically distinct regions (including frontal and parieto-occipital cerebral cortex, striatum, hippocampus, cerebellum, thalamus/midbrain, and brainstem). The parieto-occipital cortex fragment was immediately placed in cold HBSS and processed for microglia isolation. Central engraftment and microglia molecular phenotype were analyzed by flow cytometry. The frontal cortex was divided into an RNA fraction and a protein fraction, and along with the other regions, immediately flash-frozen in liquid nitrogen and stored at $-80{ }^{\circ} \mathrm{C}$ for RNA or protein analysis. Four sections of coronal slices ( $400 \mu \mathrm{m}$ per section) were obtained for ex vivo experiments from the contralateral hemibrain of each animal, and the rest of the hemibrain was then postfixed for 2 days in $4 \%$ paraformaldehyde and then placed in PBS solution containing $30 \%(\mathrm{w} / \mathrm{v})$ sucrose for 2 days at $4{ }^{\circ} \mathrm{C}$. The frozen brains were embedded in optimal cutting temperature compound, frozen in liquid isopentane, and then coronally sectioned in $40 \mu \mathrm{m}$ increments using a cryostat (Leica CM3050; Leica, Wetzlar, Germany). Slices were collected in cold cryoprotectant solution $(0.05 \mathrm{~mol} / \mathrm{l}$ sodium phosphate buffer ( $\mathrm{pH}$ 7.3), 30\% ethylene glycol, and $20 \%$ glycerol) and stored at $-20{ }^{\circ} \mathrm{C}$ until needed for immunostaining.

\section{Ex vivo Culture}

Coronal slices sectioned in $400 \mu \mathrm{m}$ were obtained from hemibrain of BM recipients or their non-transplanted controls using a Vibratome tissue slicer (Campden Instruments, Lafayette, IN). Two pieces of slices were placed into one well of a 24-well plate and cultured for $6 \mathrm{~h}$ in serum-free DMEM/ F12 medium with or without $1 \mu \mathrm{g} / \mathrm{ml}$ LPS treatment. The conditioned medium were collected and stored at $-80{ }^{\circ} \mathrm{C}$ for cytokine measurements using ELISA.

\section{Flow-Cytometric Assay}

Peripheral (non-CNS) engraftment and differentiation of $\mathrm{GFP}^{+}$donor BM-derived cells were assessed by flow cytometry of peripheral blood. Red blood cells were removed using lysis buffer (Sigma-Aldrich). Cells were then washed three times in PBS and incubated with antibodies on ice for $30 \mathrm{~min}$. Cells were fixed with $1 \%$ paraformaldehyde and then analyzed using a LSR II flow cytofluorometer (BD Biosciences, Franklin lakes, NJ). Identically processed blood from wt-GFP and wt mice were used as positive and negative controls, respectively. Peripheral blood engraftment was determined as a percentage of $\mathrm{GFP}^{+}$cells divided by the total number of nucleated cells. Multilineage differentiation of donor BM cells was determined by staining with Alexa Fluor 700-conjugated CD3 (T cells), PE-Cy7-conjugated CD19 (B cells), allophycocyanin (APC)-conjugated Gr-1 (neutrophils), and eFluor 450-conjugated CD11b (monocytes/macrophages) antibodies (eBioscience, San Diego, CA). Appropriately labeled IgG isotype control antibodies were used as negative controls.

For CNS engraftment, mononuclear cells were isolated from cerebral cortex homogenates as described previously. ${ }^{44}$ Briefly, cerebral cortex was dissociated by gentle homogenization in HBSS. The cell suspension was passed through a $70-\mu \mathrm{m}$ nylon cell strainer and centrifuged at $300 \mathrm{~g}$ for $7 \mathrm{~min}$. Supernatant was removed, and cell pellets were resuspended in 70\% isotonic percoll (GE Healthcare, Uppsala, Sweden). A discontinuous percoll density gradient was set up as follows: 70,35 , and $0 \%$ isotonic percoll. The gradient was centrifuged for $30 \mathrm{~min}$ at $1200 \mathrm{~g}$. Mononuclear phagocytes were collected from the interphase between the 70 and $35 \%$ 
percoll layers. The cells were washed and then stained with eFluor 450-conjugated CD11b and Alexa Fluor 700conjugated CD45 antibodies for $30 \mathrm{~min}$. The cell suspension was analyzed to identify the population of $\mathrm{CD} 11 \mathrm{~b}^{+} \mathrm{CD} 45^{\text {low }}$ microglia. ${ }^{45,46}$ Cerebral cortex engraftment of BM-derived microglia was determined by dividing the $\mathrm{CD}_{1} \mathrm{~b}^{+}$ $\mathrm{CD} 45^{\text {low }} \mathrm{GFP}^{+}$cell population by total $\mathrm{CD} 11 \mathrm{~b}^{+} \mathrm{CD} 45^{\text {low }}$ microglia. The assessment of cell-surface protein expression was performed using PE-Cy7-conjugated major histocompatibility complex (MHC) class II (eBioscience), PE-conjugated CD36 (LifeSpan BioSciences, Seattle, WA), and APCconjugated CD126 (Biolegend, San Diego, CA) antibodies. After washing, the cells were incubated with the fluorescentlabeled primary antibody or IgG isotype control for $30 \mathrm{~min}$ at $4{ }^{\circ} \mathrm{C}$. The expression of MHC class II, CD36 and CD126 was assessed as mean fluorescence intensity (MFI) in microglia populations. Antibody-capture beads (CompBeads; BD Biosciences) were used for single-color compensation controls for each reagent used in the study. After each control was acquired, the negative and positive populations for each color were identified. The spectral-overlap values from single color controls were automatically calculated to compensate using the BD FACSDiva ${ }^{\mathrm{TM}}$ software (BD Biosciences). For MFI, fluorochrome-conjugated isotype controls were used to quantify background, non-specific fluorescence intensity. Subtraction procedures were applied to compare the background level with the observed intensity. ${ }^{47}$ All flow-cytometry experiments were performed using a 12-color flow cytofluorometer LSR II (BD Biosciences). Data were analyzed with FlowJo software version 7.2.2 (Tree Star, Ashland, OR).

\section{ELISA}

Progranulin levels from mouse plasma and cerebral cortex were quantified using an ELISA kit (Adipogen, San Diego, CA) according to the manufacturer's protocol. To isolate protein, mouse cerebral cortex was dissected and homogenized in lysis buffer $(20 \mathrm{mM}$ Tris, $\mathrm{pH} 7.5,150 \mathrm{mM} \mathrm{NaCl}$, $1 \mathrm{mM}$ PMSF, and $0.1 \%$ Triton X-100) supplemented with protease inhibitors (Roche, South San Francisco, CA). Samples were then spun at $16000 \mathrm{~g}$ for $10 \mathrm{~min}$ at $4{ }^{\circ} \mathrm{C}$. The supernatant was collected, and the concentration of soluble protein in each sample was determined using Biorad Dc Protein Assay reagent (Bio-Rad, Hercules, CA). A total of $200 \mu \mathrm{g}$ of protein was added to $1 \times$ diluents, and samples were run in duplicate. To isolate plasma, blood was collected from the right atrium of the heart, and samples were spun at $2000 \mathrm{~g}$ for $10 \mathrm{~min}$ at $4{ }^{\circ} \mathrm{C}$. Mouse plasma was then diluted 1:500 in $1 \times$ diluent and run in duplicate. Average absorbance values were calculated for each set of duplicate standards and duplicate samples and were then used for statistical analysis. Level of acceptance for duplicates was within $20 \%$ of the mean. Unacceptable duplicates were rejected and new ones were measured. Recombinant mouse progranulin supplied with the kit was used for generating a standard curve.
Concentrations of IL- 6 and TNF- $\alpha$ in conditioned medium from slice cultures were determined by ELISA Kits (R\&D Systems, Minneapolis, MN) according to the manufacturer's instructions.

\section{Immunohistochemistry and Stereology}

Every sixth coronal section was used for immunostaining and unbiased stereological methods ( $n=4-6$ per group). Immunofluorescence staining was performed according to previously published protocols. ${ }^{48,49}$ Primary antibodies included anti-ionized calcium binding adaptor molecule 1 (Iba-1) (dilution 1:500; Wako, Richmond, VA) and antiprogranulin (dilution 1:200; R\&D Systems); species-appropriate secondary antibodies were conjugated to $\mathrm{Cy} 3$ (dilution 1:400; Jackson Immunoresearch, West Grove, PA). Prolonggold anti-fade with DAPI (Invitrogen) was used for coverslipping and nuclear counterstain. All images were captured using an FV1000 laser scanning confocal microscope (Olympus, Center Valley, PA).

To quantify $\mathrm{Iba}-1^{+}$microglia and BM-derived cells $\left(\mathrm{GFP}^{+}\right)$, sections were analyzed using unbiased stereological cell quantification using systematic random sampling. Every sixth brain section $(240 \mu \mathrm{m}$ apart $)$ was analyzed at $\times 200$ magnification using a Nikon fluorescence microscope (Melville, NY) and Stereo Investigator software version 7.52 (MBF Bioscience, Williston, VT). An optical fractionator was used with a counting frame measuring $100 \mu \mathrm{m} \times$ $100 \mu \mathrm{m}$ applied every $600 \mu \mathrm{m}$ in cerebral cortex. Cells were assessed as $\mathrm{Iba}_{-} 1^{+}, \mathrm{GFP}^{+}$, or $\mathrm{Iba}_{-}{ }^{+}$and $\mathrm{GFP}^{+}$double immunopositive.

\section{RT-PCR and Quantitative Real-time PCR}

Total RNA was extracted from mouse cortex with RNeasy (Qiagen, Valencia, CA) according to the manufacturer's suggestions. Progranulin expression was determined via RT-PCR using RNA from cerebral cortex of wt-GFP $\rightarrow$ wt, wt-GFP $\rightarrow \mathrm{Grn}^{-1-}$, wt or $\mathrm{Grn}^{-1-}$ mice as templates, and amplified with a primer (forward, 5'-CTGTCGTGTGCCC TGATGCTAAG-3'; reverse, 5'-CCCCAGTCCCCAGAATTG AGTTTG- $3^{\prime}$ ). Mouse $\beta$-actin was used as a control.

To quantify progranulin or cytokine expression in cerebral cortex, real-time RT-PCR was performed in a model 7300 real-time PCR system (Applied Biosystems, Foster City, CA) using iTaq Universal SYBR Green assay (Bio-Rad, Hercules, $\mathrm{CA})$. One $\mu \mathrm{g}$ of total RNA was reverse-transcribed using a RETROscript kit (Bio-Rad). The cDNA synthesized from total RNA was diluted 20-fold with DNase-free water, and each cDNA sample was independently tested three times. The primer sequences are Grn (forward, 5'-GACACATGGCCTA GAATAAC- $3^{\prime}$; reverse, 5'-AAGACACACCCTTAGAGAAC-3'); CCL2 (forward, 5'-CAAGATGATCCCAATGAGTAG-3'; reverse, 5'-TTGGTGACAAAAACTACAGC-3'); CX3CL1 (forward, 5'-CTTCCATTTGTGTACTCTGC-3'; reverse, 5'-ACTCCTGG TTTAGCTGATAG-3'); Macrophage migration inhibitory factor (MIF) (forward, 5'-GGGTCTACATCAACTATTACG-3'; 
reverse, 5'-GGATAAACACAGAACAC TACG-3'); CXCL10 (forward, 5'-AAAAAGGTCTAAAAGGGCTC-3'; reverse, $5^{\prime}$-AATTAGGACTAGCCATCCAC- $3^{\prime}$ ). Mouse $\beta$-actin expression was used as an endogenous control (forward, $5^{\prime}$-GAT GTATGAAGGCTTTGGTC-3'; reverse, 5'-TGTGCACTTTTA TTGGTCTC- $\left.3^{\prime}\right)$. Cycling conditions for real-time PCR were $95^{\circ} \mathrm{C}$ for $20 \mathrm{~s}$, followed by 40 cycles at $95^{\circ} \mathrm{C}$ for $1 \mathrm{~s}$ and $60{ }^{\circ} \mathrm{C}$ for $20 \mathrm{~s}$. Quantitative PCR was performed according to the guidelines provided by Applied Biosystems. The comparative cycle threshold (CT) method ( $\Delta \Delta \mathrm{CT}$ quantitation) was used to assess the difference between samples. Quantitative data analysis followed the suggestions of the manufacturer.

\section{Statistical Analysis}

Results are expressed as mean \pm standard error of the mean (s.e.m.). Statistical analysis was performed by the unpaired Student's t-test or one- or two-way analysis of variance as indicated. Post hoc testing used the Bonferroni method. Statistical significance was assumed if $P<0.05$. All statistical analyses were performed using GraphPad Prism software version 5.03 (San Diego, CA).

\section{RESULTS}

\section{Partial Reconstitution of Progranulin in Periphery and Cerebral Cortex of $\mathrm{Grn}^{-I-}$ Mice}

BMT-derived cells migrate into the CNS where they adopt a microglial phenotype and partially replace existing monocyte-lineage cells in irradiated wt-recipient mice. ${ }^{48,50-52} \mathrm{We}$ transplanted erythrocyte-depleted whole BM from wt-GFP donor mice into 2-month-old wt $(w t-G F P \rightarrow w t)$ or agematched $\mathrm{Grn}^{-/-}\left(\mathrm{wt}-\mathrm{GFP} \rightarrow \mathrm{Grn}^{-/-}\right)$recipient mice $24 \mathrm{~h}$ after myeloablative $(10.5 \mathrm{~Gy})$ total body irradiation. All recipients survived until they were euthanized 5 months post BMT. Flow cytometry (Figure 1a) showed essentially complete marrow replacement since $>96 \%$ of blood mononuclear cells were $\mathrm{GFP}^{+}$(donor-derived). The extent of peripheral engraftment did not differ by recipient genotype (Figure 1b): wt-GFP $\rightarrow$ wt, $96.4 \pm 0.3 \%$; wt-GFP $\rightarrow \mathrm{Grn}^{-1-}$, $96 \pm 0.7 \%$. Using lineage-specific antibodies, we compared the proportion of $\mathrm{T}$ cells, B cells, neutrophils, and monocytes/ macrophages in the blood of BMT-recipient mice (Figure 1c) and found no significant effect of recipient $G m$ genotype.

We next determined whether recipient genotype influenced the engraftment of wt cells into cerebral cortex. Mononuclear cells from cerebral cortex (perfused with icecold PBS) were isolated for flow cytometry and microglia $\left(\mathrm{CD} 11 \mathrm{~b}^{+} \mathrm{CD} 45^{\text {low }}\right)$ probed for expression of GFP (Figure 1d). In contrast to peripheral leukocyte engraftment, significantly less BMT-derived microglia engrafted in cerebral cortex of $\mathrm{Grn}^{-1-}$ recipients $(10.6 \pm 2.3 \%)$ than in wt recipients $(22.8 \pm 2.8 \%$, Figure 1e, $P<0.01)$. We investigated four potential mediators of reduced engraftment of BMT-derived cells in $\mathrm{Grn}^{-1-}$ cerebral cortex (Figure 1f): CXCL10 (IP-10), CCL2 (MCP-1), CX3CL1 (fractalkine/neurotactin), and macrophage migration inhibitory factor (MIF). CXCL10 cerebral cortical mRNA level was increased about twofold in wt-GFP $\rightarrow$ wt mice without significant change in wt-GFP $\rightarrow$ $\mathrm{Grn}^{-1-}$ mice compared with non-transplanted controls. In contrast, similarly increased expression of CCL2 was observed in the cerebral cortex of both recipient groups compared with their non-transplanted controls. Finally, qPCR analysis of cerebral cortical CX3CL1 and MIF mRNA levels showed that each was unchanged in recipient cerebral

Figure 1 Engraftment of GFP ${ }^{+}$cells in peripheral blood and CNS of BMT recipients. (a) Representative flow-cytometric histograms showing the expression of $\mathrm{GFP}^{+}$cells in blood 5 months after transplantation with BM cells into lethally irradiated wt (wt-GFP $\rightarrow$ wt ) or Grn ${ }^{-/-}$(wt-GFP $\rightarrow$ Grn $^{-/-}$) mice. A C57BL6;GFP mouse (GFP) and a C57BL6 mouse (wt) were used as positive and negative controls, respectively. (b) Percent peripheral engraftment was calculated by comparing GFP ${ }^{+}$leukocytes with total leukocytes using flow cytometry, and revealed nearly complete peripheral engraftment with no significant host genotype differences detected. (c) Flow-cytometric analysis of peripheral blood comparing the proportion of T cells, B cells, neutrophils, and monocytes/macrophages in BMT-recipient mice and controls. Data revealed no significant influence of host genotype on the percentage of respective cell populations. Data are expressed as mean \pm s.e.m. Six animals per group were analyzed. (d) Mononuclear cells were isolated using percoll gradient from rapidly dissected cerebral cortex from wt-GFP $\rightarrow$ wt and wt-GFP $\rightarrow$ Grn ${ }^{-/-}$chimeras five months post transplantation after transcardial perfusion with ice-cold PBS. Representative flow-cytometric histogram of GFP fluorescence (x axis) in CD11b ${ }^{+}$CD45 ${ }^{\text {low }}$ gate is shown for population of recipient $\left(\mathrm{GFP}^{-}\right)$vs donor $\left(\mathrm{GFP}^{+}\right)$microglia from cerebral cortex. Negative control graph shows the histogram of a C57BL6 mouse (wt-upper left). Positive control graph shows the histogram of a C57BL6;GFP mouse (GFP-upper right). (e) Flow-Cytometric analysis showed engraftment of BMT-derived microglia (GFP ${ }^{+} \mathrm{CD} 11 \mathrm{~b}^{+} \mathrm{CD} 45^{\text {low }}$ ) was decreased in wt-GFP $\rightarrow$ Grn ${ }^{-/-}$chimeras compared with wt-GFP $\rightarrow$ wt mice. ${ }^{* *} P<0.01$, unpaired Student's t-test. (f) Quantitative real-time PCR analysis of CXCL10 and CCL2 mRNA expression in cerebral cortex of BMT recipients and the non-transplanted controls. Expression of CCL2 was below the limit of detection in cortex of wt or Grn ${ }^{-1-}$ non-transplanted mice. Expression of CCL2 in cortex was induced in wt-GFP $\rightarrow$ wt and wt-GFP $\rightarrow G r n^{-1}$ mice, but no significant difference was observed between the two types of chimeras. CXCL10 mRNA expression was increased in wt-GFP $\rightarrow$ wt mice compared with wt mice $\left({ }^{*} P<0.05\right)$, whereas no significant difference was observed between wt-GFP $\rightarrow G r n^{-1-}$ mice and $\mathrm{Grn}^{-1-}$ mice. One-way ANOVA was performed using the Bonferroni post hoc test. Data are expressed

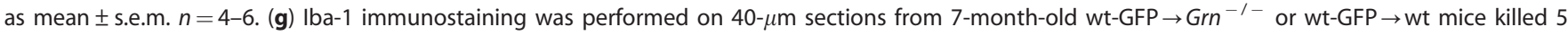
months post BMT. Merged images confirm that GFP ${ }^{+}$cells are also uniformly lba- ${ }^{+}$(yellow) and therefore of donor origin, whereas others represent

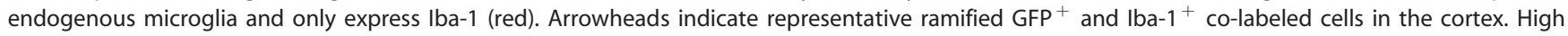
magnification images of GFP ${ }^{+}$(green) and lba-1 ${ }^{+}$immunoreactivity (red) for microglia revealed predominantly ramified morphology in both groups with no clear differences between groups. Scale bars: $50 \mu \mathrm{m} ; 10 \mu \mathrm{m}$ (high magnifications). (h) Unbiased stereological quantitation of BMT-derived microglia engraftment in the cortex of chimeric mice reveals reduced engraftment in wt-GFP $\rightarrow G r n^{-1-}$ mice compared with wt-GFP $\rightarrow$ wt mice. ${ }^{*} P<0.01$, unpaired Student's t-test. Individual mice and the mean \pm s.e.m per group are shown. 
cortex compared with non-transplanted controls (data not shown).

Confocal microscopic examination of cerebral cortex showed that donor BMT-derived $\left(\mathrm{GFP}^{+}\right)$parenchymal cells exhibited a ramified phenotype and were decorated with the microglial marker Iba-1 in wt-GFP $\rightarrow$ wt and in wt-GFP $\rightarrow \mathrm{Grn}^{-1-}$ chimeric mice (Figure 1g). Unbiased stereological quantitation of endogenous and donor-derived microglia confirmed our flow-cytometric results since there was a significant $(P<0.01)$ reduction in donor-cell engraft- ment in the cerebral cortex of wt-GFP $\rightarrow \mathrm{Grn}^{-1-}$ mice compared with wt-GFP $\rightarrow$ wt mice (Figure $1 \mathrm{~h}$ ).

Progranulin in plasma and cerebral cortex from $\mathrm{Grn}^{-1-}$ mice was below the limit of detection by ELISA (Figures 2a and $b$ ). There was no difference in plasma or cerebral cortical progranulin level between wt and wt-GFP $\rightarrow$ wt mice. Average progranulin levels in plasma of wt-GFP $\rightarrow \mathrm{Grn}^{-1-}$ mice were $23 \%$ of wt-GFP $\rightarrow$ wt mice, while average progranulin level in cerebral cortex of wt-GFP $\rightarrow \mathrm{Grn}^{-1-}$ mice was $8 \%$ of wt-GFP $\rightarrow$ wt mice (Figures $2 \mathrm{a}$ and b). Progranulin a

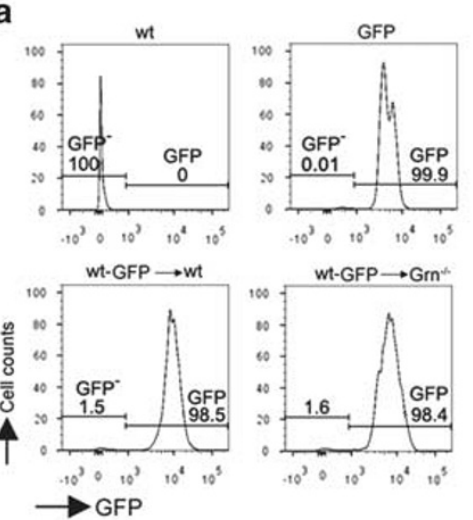

d

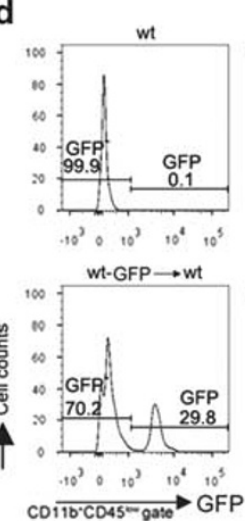

g
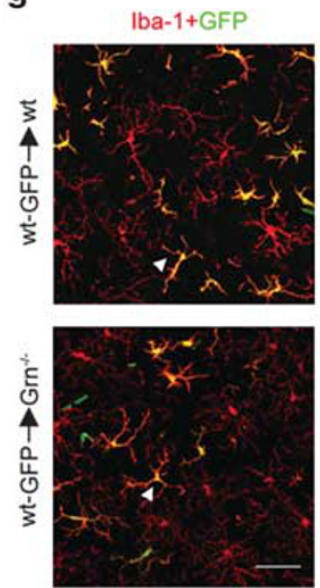

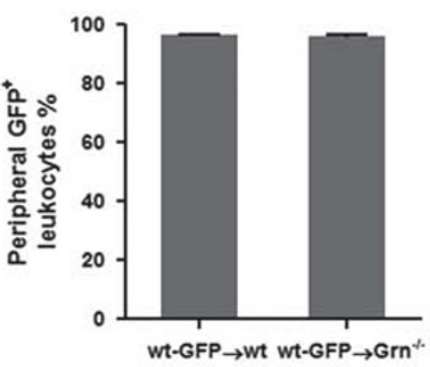

e
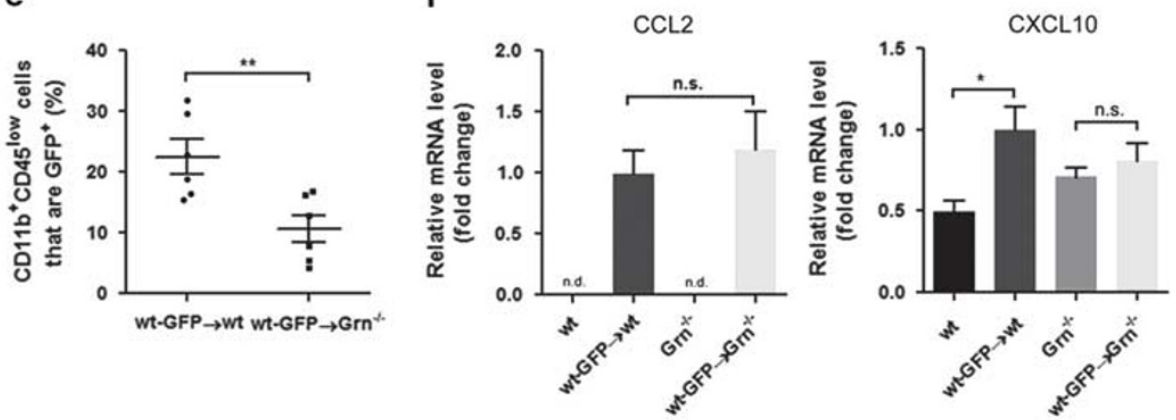

Iba-1
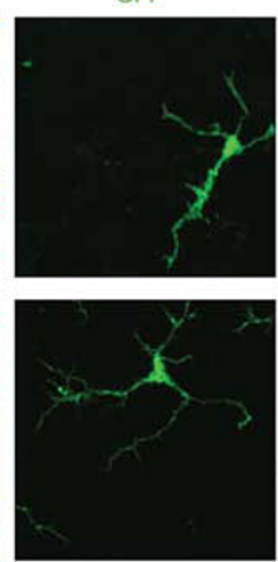

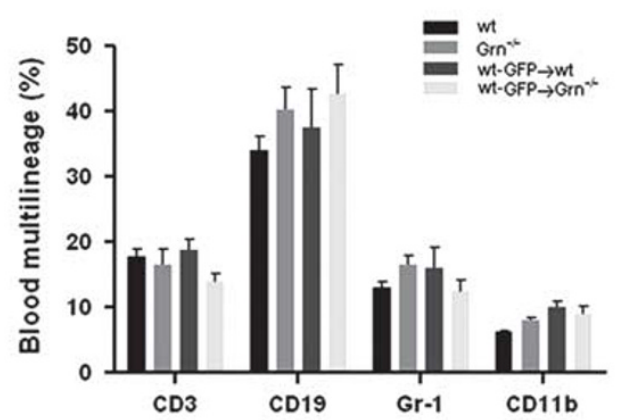

f

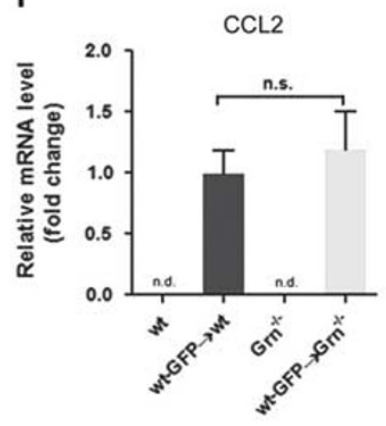

C

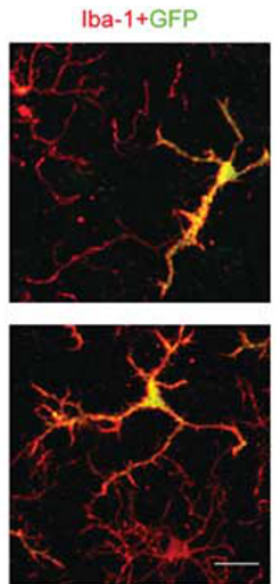

h

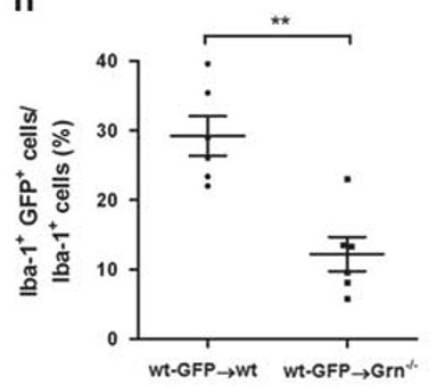

Figure 1 For caption please refer page 1227. 


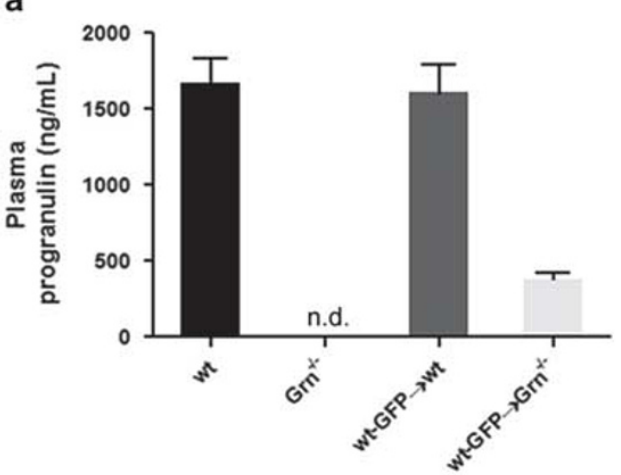

C

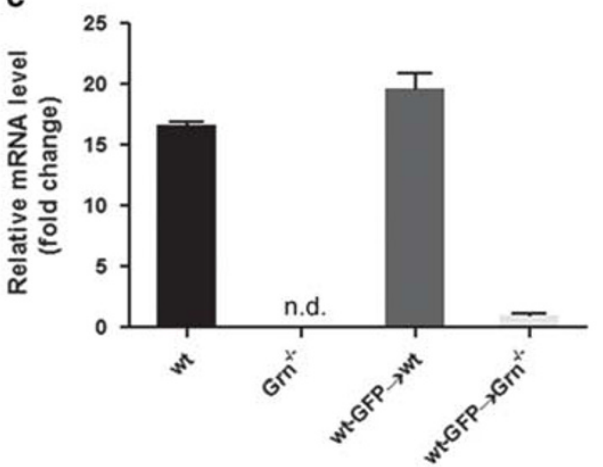

e

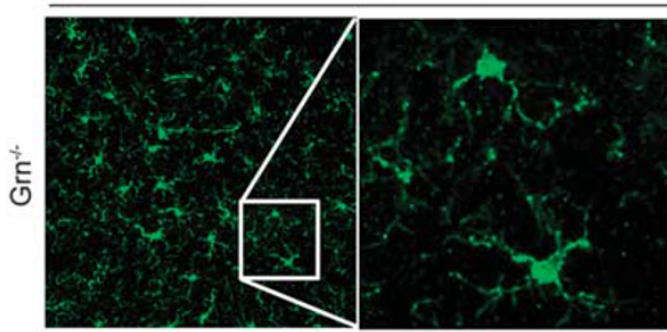

GFP+Grn

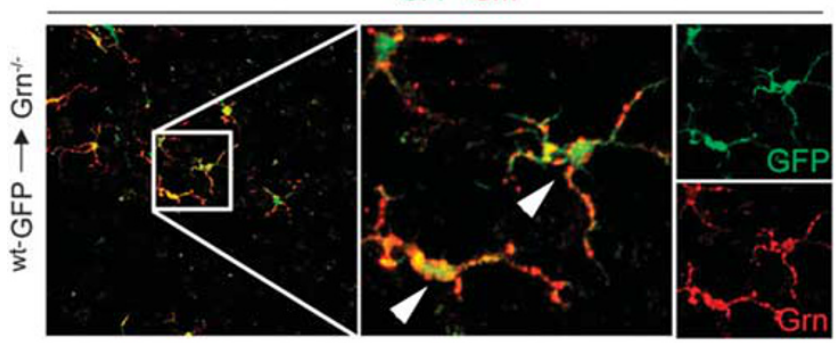

b

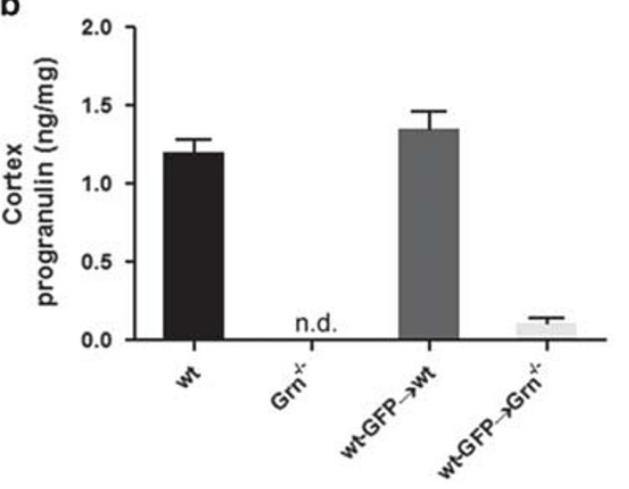

d

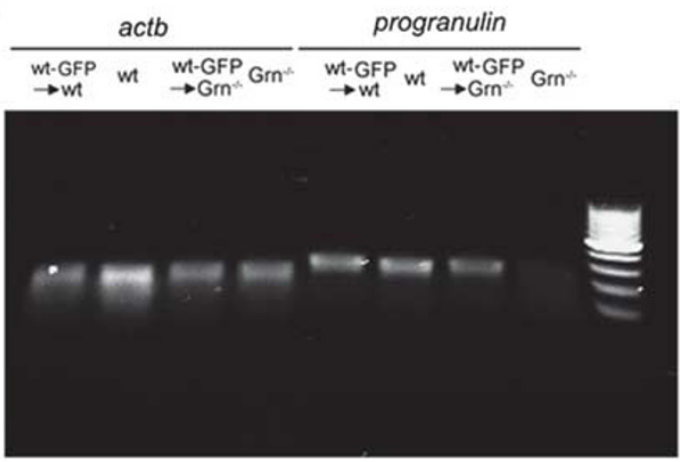

Iba-1+Grn

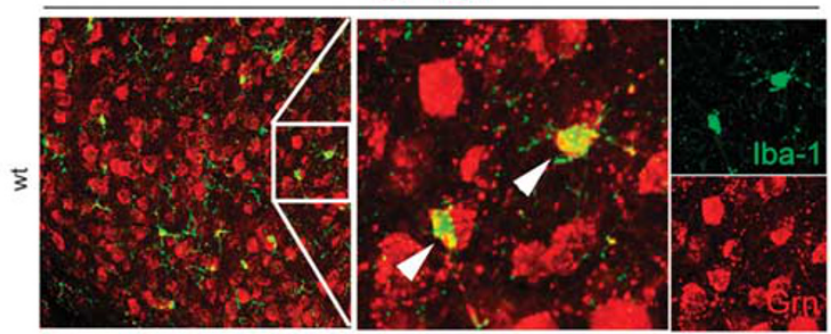

GFP+Grn

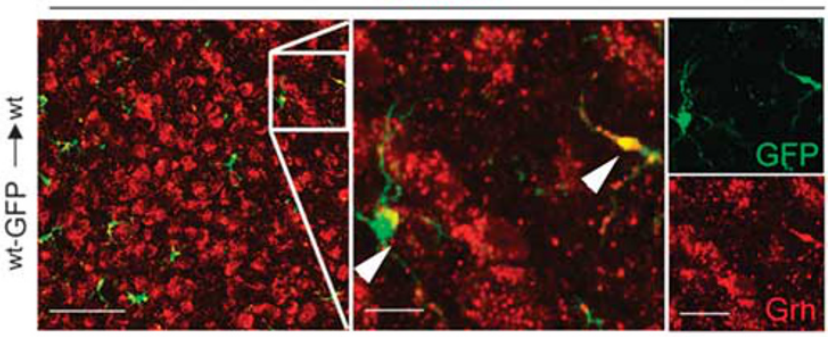

Figure 2 Detection of progranulin levels in BMT recipients. (a) Progranulin protein levels $(\mathrm{ng} / \mathrm{ml}$ ) in the plasma of chimeric mice 5 months post BMT and non-transplanted controls were measured by ELISA. No progranulin was detected in plasma of $\mathrm{Grn}^{-/-}$mice. The progranulin level in wt-GFP $\rightarrow$ $\mathrm{Grn}^{-1-}$ mice was $23 \%$ of the level of wt-GFP $\rightarrow$ wt mice. No difference was observed between wt-GFP $\rightarrow$ wt mice and wt non-transplanted control mice. (b) Cortex Tris-HCl buffer lysates from chimeric mice 5 months post BMT were subjected to ELISA for progranulin. The non-transplanted wt and $\mathrm{Grn}^{-1-}$ mice served as controls. The progranulin level $(\mathrm{ng} / \mathrm{mg})$ in wt-GFP $\rightarrow G r n^{-1-}$ was $8 \%$ of the level (ng/mg) of wt-GFP $\rightarrow$ wt mice. No difference was found between wt control mice and wt-GFP $\rightarrow$ wt mice. (c) Progranulin mRNA in cerebral cortex from BMT-recipient mice and their nontransplanted controls were measured by real-time PCR analysis. Grn transcripts were not detected in non-transplanted $\mathrm{Grn}^{-1-}$ but were identified in wt-GFP $\rightarrow \mathrm{Grn}^{-1-}$ mice. (d) Agarose gel assay of progranulin PCR production compared with beta-actin (actb) as a control. (e) Progranulin and Iba-1 immunostaining was performed on 40- $\mu \mathrm{m}$ sections from 7-month-old wt or $\mathrm{Grn}^{-1-}$ mice killed 5 months post BMT or their age-matched nontransplanted controls. Representative images from $\mathrm{Grn}^{-1-}$ mouse demonstrate no progranulin immunoreactivity, whereas co-immunostaining of progranulin (red) and Iba-1 (green) was observed in cerebral cortex of wt controls, confirming microglial expression of progranulin. BMT-derived (GFP ${ }^{+}$) microglia (green) express progranulin (red) in wt-GFP $\rightarrow \mathrm{Grn}^{-/-}$mice. Images of areas of interest (white squares) are shown at increasing magnifications (scale bars: $20 \mu \mathrm{m}$ ). Scale bars: $50 \mu \mathrm{m}$. 
transcription was detected in cerebral cortex of wt-GFP $\rightarrow$ $\mathrm{Grn}^{-1-}$ mice (Figures $2 \mathrm{c}$ and d). These data indicate that cellular sources other than BMT-derived cells contribute to plasma and cerebral cortical progranulin concentration. BMT led to in situ transcription of progranulin in $\mathrm{Grn}^{-1-}$ cerebral cortex, suggesting that BMT-derived microglia are able to partially reconstitute CNS progranulin.

To further evaluate for this possibility, we performed immunofluorescent double labeling with anti-progranulin antibody and specific markers for microglia Iba-1. Abundant $\mathrm{Iba}^{+}$microglia were present in $\mathrm{Grn}^{-1-}$ mice but these cells were uniformly negative for progranulin immunoreactivity (Figure 2e, upper, left). As expected, wt mice demonstrated strong and diffuse neuronal and microglial progranulin immunoreactivity (Figure 2e, upper, right). In BMT-recipient mice, $\mathrm{GFP}^{+}$ramified cells in cerebral cortex of wt-GFP $\rightarrow \mathrm{Grn}^{-1-}$ mice co-labeled with progranulin (Figure 2e, lower, left), demonstrating that BM-derived cells express progranulin following engraftment in brain where they adopt a microglia phenotype. We verified the presence of $\mathrm{GFP}^{+}$ramified cells in cerebral cortex of wt-GFP $\rightarrow \mathrm{wt}$ mice that co-labeled with progranulin (Figure 2e, lower, right); in this case we also observed expected extensive neuronal progranulin immunoreactivity, which presumably represents the non-BMT-derived source of progranulin from Figure $2 b$.

\section{Immune Modulation Ex Vivo}

Prominent neuropathological changes and behavioral differences are described in $\mathrm{Grn}^{-1-}$ mice older than those in our study; however, alterations in innate immune function occur much earlier. To evaluate the functional consequences of BMT in $\mathrm{Grn}^{-1-}$ mice, we assayed the immune modulatory activity of progranulin ex vivo using cerebral slice cultures. In these experiments, we stimulated innate immune reaction by administering LPS (activator of Toll-like receptor (TLR) 4), and assessed innate immune response in microglia by measuring TNF- $\alpha$ levels in cell culture media and in astrocytes by measuring IL- 6 in culture media. ${ }^{53-55}$ TNF- $\alpha$ was undetectable in conditioned medium from unstimulated cerebral coronal slices from control and BMT-recipient wt mice. In contrast, TNF- $\alpha$ was present at low concentrations in conditioned medium from unstimulated $\mathrm{Grn}^{-1-}$ and wt-GFP $\rightarrow \mathrm{Grn}^{-1-}$ slices, consistent with a net immunosuppressive role of progranulin at baseline ${ }^{22}$ (Figure 3). TNF- $\alpha$ expression was increased significantly in cerebral slices exposed to LPS compared with vehicle for all groups (Figure $3, P<0.0001)$. LPS-induced TNF- $\alpha$ secretion was significantly increased in $\mathrm{Grn}^{-1-}$ cerebral slices compared with wt slices (Figure 3, $P<0.0001$ ), confirming an exaggerated innate immune response in the absence of progranulin. ${ }^{13,22}$ This pro-inflammatory effect was significantly mitigated by wt BMT; cerebral slices from wt-GFP $\rightarrow$ $\mathrm{Grn}^{-1-}$ mice had an average 38\% reduction in LPS-induced TNF- $\alpha$ expression compared with slices from non-

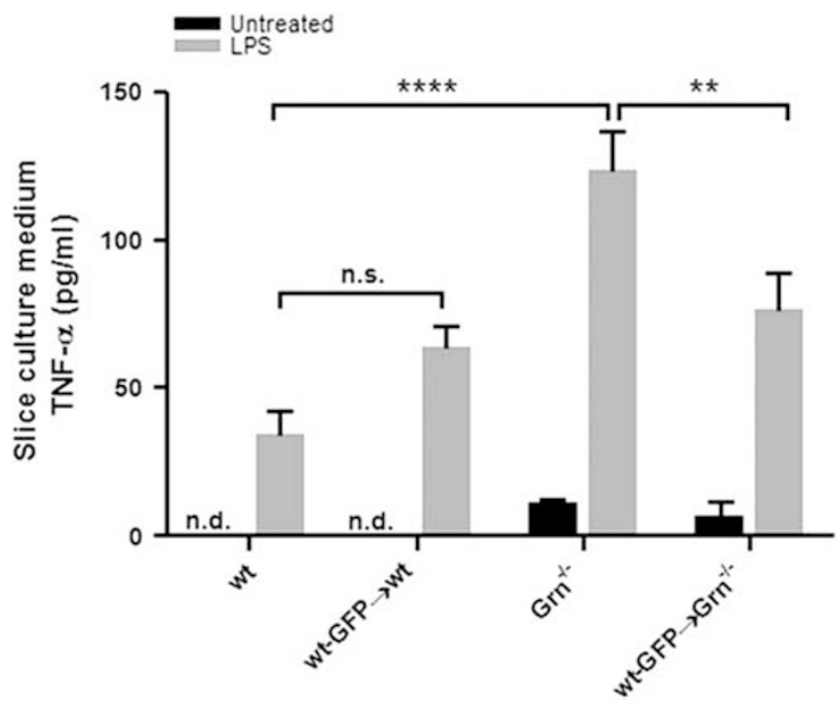

Figure 3 Alteration in ex vivo inflammatory responses to LPS in BMT recipients.Hemibrain slices were incubated with serum-free DMEM/F12 medium with or without LPS. Conditioned media collected $6 \mathrm{~h}$ after adding $1 \mu \mathrm{g} / \mathrm{ml}$ LPS was analyzed by ELISA. TNF- $\alpha$ expression was significantly increased in conditioned media from slices treated with LPS. Two-way ANOVA had ${ }^{* * * *} P<0.0001$ for untreated vs LPS. TNF- $\alpha$ expression in response to LPS was significantly higher in $\mathrm{Grn}^{-1}$ mice compared with wt mice $\left.{ }^{* * * *} P<0.0001\right)$, but was significantly decreased in wt-GFP $\rightarrow \mathrm{Grn}^{-1-}$ brain slices in comparison with $\mathrm{Grn}^{-1-}$ brain slices $\left({ }^{*} P<0.01\right.$, Bonferroni corrected posttest). No significant difference in TNF- $\alpha$ expression in response to LPS was found between wt and wt-GFP $\rightarrow$ wt mice. All results are expressed as means \pm s.e.m, $n=4-6$.

transplanted $\mathrm{Grn}^{-1-}$ mice (Figure 3, P<0.01), indicating BMT-mediated partial restoration of progranulin immunomodulation that, in this slice preparation from a perfused brain, is interpreted as a direct result of wt microglia engraftment. IL- 6 was detectable in conditioned medium from all slice cultures exposed to vehicle and its concentration was not significantly different among the four groups (not shown). LPS exposure significantly increased IL-6 concentration in all groups $(P<0.0001)$; however, there was no significant difference in IL-6 concentration between $\mathrm{Grn}^{-1-}$ slices $(33.2 \pm 7.8 \mathrm{pg} / \mathrm{ml})$ and wt-GFP $\rightarrow \mathrm{Grn}^{-1-}$ slices $(42.1 \pm 7.5 \mathrm{pg} / \mathrm{ml})$ in response to LPS. IL-6 in slice cultures is primarily produced and secreted by astrocytes, providing further support for neuroimmunomodulation in $\mathrm{Grn}^{-1-}$ that is BMT-derived microglia mediated.

\section{Immune Modulation In Vivo}

CD126 is a receptor for IL-6, and dysregulated production of IL-6 and CD126 is implicated in innate immune modulation. ${ }^{53,56-58} \mathrm{CD} 36$ is a key microglial receptor for phagocytosis ${ }^{59,60}$ and innate immune response. ${ }^{61}$ MHC class II is critically involved in microglia innate immune regulation and antigen presentation and is increased in BMT-derived microglia. ${ }^{48,62}$ We hypothesized that progranulin deficiencyrelated alterations in microglia molecular phenotype would 
be mitigated in BMT-derived cells. To test this, we measured microglia CD126, CD36, and MHC class II protein expression using flow cytometry of cortical microglia from control or BMT-recipient wt and $\mathrm{Grn}^{-1-}$ mice. Two-way ANOVA revealed that CD126 (Figure 4a) and CD36 (Figure $4 \mathrm{~b}$ ) MFIs were significantly different for genotype (wt vs $\mathrm{Grn}^{-1-}, P<0.01$ for CD126, $P<0.001$ for CD36) with no significant difference for transplant $v s$ non-transplant but a significant interaction $(P<0.05$ for CD126, $P<0.01$ for CD36) between these two dimensions. Bonferroni-corrected posttests showed that wt $v \mathrm{Grn}^{-1-}$ were significantly different $(P<0.01$ for CD126, $P<0.001$ for CD36) with MFI of both CD126 and CD36 increased in $\mathrm{Grn}^{-1-}$ compared with wt. Importantly, the two transplanted groups were not significantly different for either CD126 or CD36 MFI. Together, these data demonstrate an immunomodulatory effect of progranulin or its proteolytic products on cerebral cortical microglia in vivo that is completely restored by BMT.

To determine whether the restored immunomodulatory effect of progranulin by BMT was mediated by donor or recipient microlglia, we analyzed CD126 (Figure 4c) and CD36 (Figure 4d) MFIs using two-way ANOVA for cerebral cortical microglia distinguished as either recipient $\left(\mathrm{GFP}^{-}\right)$ or BMT-derived $\left(\mathrm{GFP}^{+}\right)$for the two transplanted groups. CD126 MFI was significantly different for recipient $v s$ donor microglia $(P<0.01)$ but not for treatment and there was not a significant interaction term; Bonferroni-corrected posttests showed that CD126 MFI was significantly greater in recipient $v s$ donor microglia for the wt-GFP $\rightarrow$ wt $(P<0.01)$ but not different in the wt-GFP $\rightarrow \mathrm{Grn}^{-1-}$ mice, indicating a potential recipient-dependent reduction in CD126 levels in donor microglia. CD36 MFI was significantly different for donor $v s$ recipient microglia $(P<0.001)$, wt-GFP $\rightarrow$ wt $v s$ wt-GFP $\rightarrow \mathrm{Grn}^{-1-}(P<0.001)$ and there was a significant interaction between genotype and treatment $(P<0.01)$. Bonferroni-corrected posttest showed that this significant interaction resided in significantly greater CD36 MFI in donor $v s$ recipient microglia in wt-GFP $\rightarrow \mathrm{Grn}^{-1-}$ mice $(P<0.001)$ but no difference in CD36 MFI in donor vs recipient microglia in wt-GFP $\rightarrow$ wt mice. These results show that, at least for microglial CD36 MFI, the effect of lack of proganulin on recipient microglia could be completely reversed in donor microglia.

We measured MHC class II expression and found that, unlike CD126 and CD36, there was no significant difference between non-transplanted wt and $\mathrm{Grn}^{-1-}$ mice. MFI for wt mice was $651 \pm 386$ and for $\mathrm{Grn}^{-1-}$ mice was $1327 \pm 248$ $(n=4-6 ; P>0.05)$. As expected, MHC class II MFI on total microglia was significantly increased in wt-GFP $\rightarrow \mathrm{wt}$ mice $^{48,62}(P<0.05)$ with similar increase in MHC class II MFI in wt-GFP $\rightarrow \mathrm{Grn}^{-1-}$ mice $(P<0.01)$ such that the MHC class II total microglia MFI was not different between the two BMT groups (not shown). In spite of this, donor and recipient MHC class II was also analyzed to determine whether brain progranulin deficiency differentially modulated donor or recipient MHC class II expression. MHC class II MFI was at the limit of detection for recipient $\left(\mathrm{GFP}^{-}\right)$microglia in both wt-GFP $\rightarrow$ wt and wt-GFP $\rightarrow$ $\mathrm{Grn}^{-1-}$ mice (Figure 4e), which most likely underlies a lack of genotype differences in overall MHC II expression. Donorderived $\left(\mathrm{GFP}^{+}\right)$microglia MHC II was significantly increased for both transplant groups (Figure $4 \mathrm{e}, P<0.01$ for wt-GFP $\rightarrow$ wt mice and $P<0.001$ for wt-GFP $\rightarrow \mathrm{Grn}^{-1-}$ ). MHC class II MFI on BMT-derived microglia was significantly greater in wt-GFP $\rightarrow \mathrm{Grn}^{-1-}$ mice than in wt-GFP $\rightarrow$ wt mice (Figure $4 \mathrm{e}, P<0.001$ ), consistent with altered immunomodulation of donor microglia in $\mathrm{Grn}^{-1-}$ mice in a similar manner to CD36.

Next, we assessed microglia cell densities in control and transplant recipients to determine whether progranulin deficiency affects total microglia and whether this was altered with wt BMT. Grn ${ }^{-1-}$ mice display age-dependent cerebral microgliosis that has been reported at 7 months of age ${ }^{63}$ and reaches a peak at around 12 months of age. ${ }^{37,64}$ We performed unbiased stereological analysis of total Iba- ${ }^{+}$cell density in cerebral cortex (Figure 5a) and analyzed the data by two-way ANOVA comparing non-transplanted $v s$ BMT in wt $v s \mathrm{Grn}^{-1-}$ mice (Figure 5b). Our results confirmed a significant $\sim 20 \%$ increase in Iba- ${ }^{+}$cells in $\mathrm{Grn}^{-1-}$ compared with wt non-transplanted mice at 7 months of age ( 5 months post BMT, $P<0.05$ ). BMT had a significant effect on microglia density $(P<0.001)$ with Bonferroni-corrected posttests showing significantly reduced microglia density both in wt-GFP $\rightarrow \mathrm{Grn}^{-1-}$ mice compared with $\mathrm{Grn}^{-1-}$ mice $(P<0.001)$ and in wt-GFP $\rightarrow$ wt mice compared with wt mice $(P<0.01)$. There also was a significant interaction term $(P<0.05)$. Although we are unsure of the mechanism by which wt-GFP $\rightarrow$ wt reduced cerebral cortical microglia density compared with wt, the major finding of this experiment is that wt-GFP $\rightarrow \mathrm{Grn}^{-1-}$ completely erases progranulin deficiency-dependent cerebral cortical microgliosis.

\section{DISCUSSION}

Progranulin replacement therapy represents a potentially attractive approach to prevent or treat forms of FTLD-TDP caused by GRN haploinsufficiency. Such efforts are complicated by the many, sometimes functionally antagonistic, actions of progranulin and its bioactive proteolytic products. Indeed, we are unaware of any successful small molecule program to enhance progranulin expression or activity, ${ }^{65}$ although one group has published on small molecule inhibitors of sortilin that increase extracellular concentrations of progranulin in cell culture. ${ }^{40}$ Another group recently reported that viral-mediated transfer of progranulin into substantia nigra partially protected from 1-methyl-4-phenyltetrahydropyridine dopaminergic neurotoxicity. ${ }^{66}$ Since BM-derived cells, including microglia, express and secrete progranulin, here we tested the hypothesis that BMT would partially replace progranulin and its activity in cerebral 

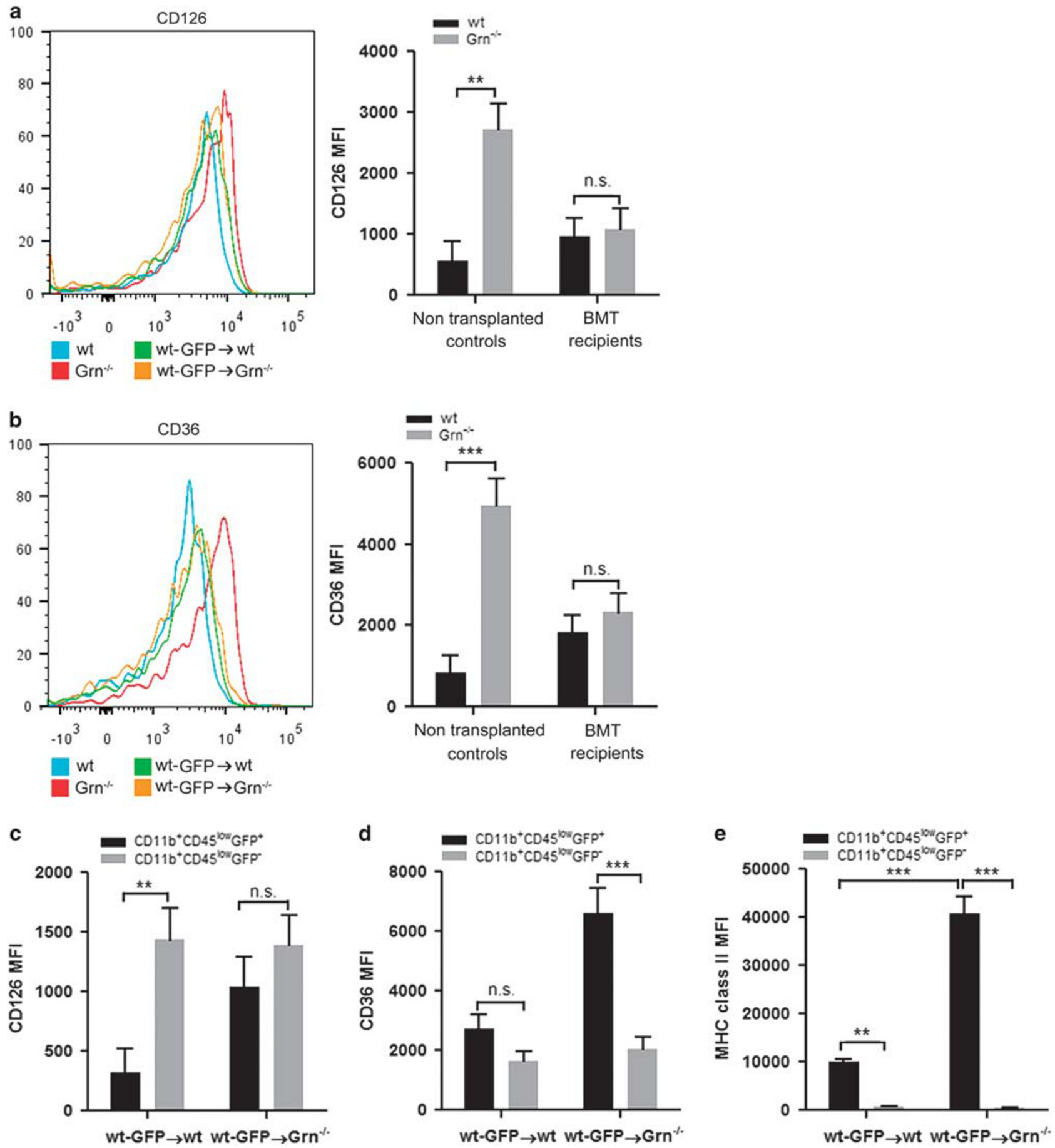

Figure 4 Microglia molecular phenotype modulation in BMT-recipient mice. Mononuclear cells in cerebral cortex were isolated using percoll gradient and subjected to flow-cytometric analysis for microglial expression of innate immune effector molecules. (a) Representative histogram of flowcytometric analysis for $\mathrm{CD} 126$ expression in $\mathrm{CD} 11 \mathrm{~b}^{+} \mathrm{CD} 45^{\text {low }}$ microglia (left panel). CD11b ${ }^{+} \mathrm{CD} 45^{\text {low }}$ microglial CD126 mean fluorescence intensity (MFI) was significantly increased in $\mathrm{Grn}^{-1-}$ mice compared with wt mice (**P<0.01). No significant difference was observed between transplanted groups (right panel). (b) Representative histogram of flow-cytometric analysis for CD36 expression on $\mathrm{CD} 11 \mathrm{~b}^{+} \mathrm{CD} 45^{\text {low }}$ microglia population (left panel). CD36 expression on microglia $\left(\mathrm{CD} 11 \mathrm{~b}^{+} \mathrm{CD} 45^{\text {low }}\right)$ was significantly increased in $\mathrm{Grn}^{-1-}$ mice compared with wt mice $\left({ }^{* * *} \mathrm{P}<0.001\right)$ with no significant difference for the two transplanted groups (right panel). (c) CD126 expression was significantly lower in donor $\left(\mathrm{CD} 11 \mathrm{~b}^{+} \mathrm{CD} 45^{\text {low }} \mathrm{GFP}{ }^{+}\right.$) vs recipient $\left(\mathrm{CD} 11 \mathrm{~b}^{+} \mathrm{CD} 45^{\text {low }} \mathrm{GFP}^{-}\right)$microglia in wt-GFP $\rightarrow$ wt mice $\left.{ }^{* *} \mathrm{P}<0.01\right)$, but not different in the wt-GFP $\rightarrow \mathrm{Grn}^{-1-}$ mice. (d) CD36 MFI was significantly greater in donor vs recipient microglia in wt-GFP $\rightarrow G n^{-1-}$ mice $\left({ }^{* *} P<0.001\right)$ but no difference in donor vs recipient microglia in wt-GFP $\rightarrow$ wt mice. (e) MHC class II expression on donor-derived microglia was significantly increased for both wt-GFP $\rightarrow$ wt mice $(* * P<0.01)$ and wt-GFP $\rightarrow G r n^{-\prime-}$ mice $(* * * P<0.001)$. MHC class II MFI on donor microglia was significantly greater in wt-GFP $\rightarrow G r^{-1-}$ mice than in wt-GFP $\rightarrow$ wt mice (***P<0.001). All results are expressed as means \pm s.e.m., $n=4-6$. Two-way ANOVA was performed using the Bonferroni post hoc test. 
a

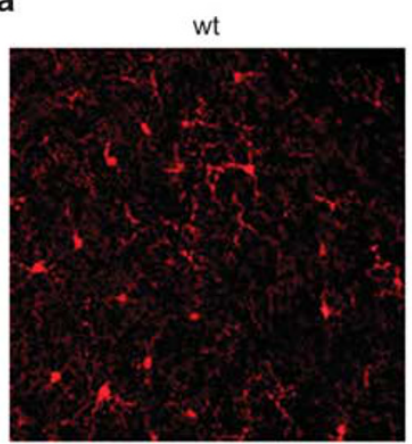

$w t-G F P \rightarrow w t$
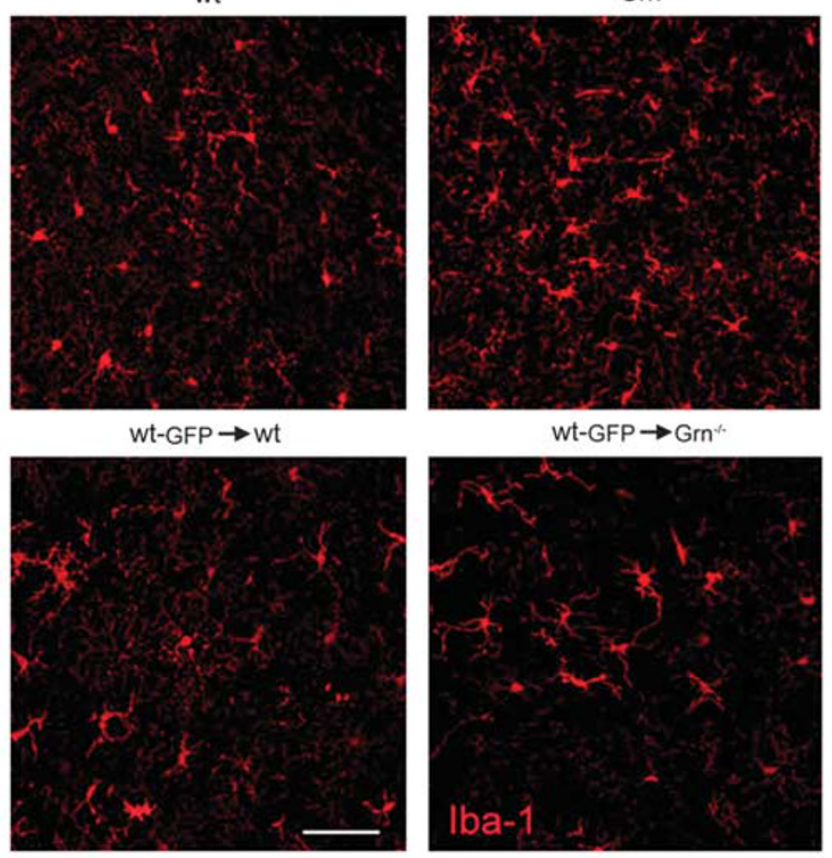

wt-GFP $\rightarrow$ Grn

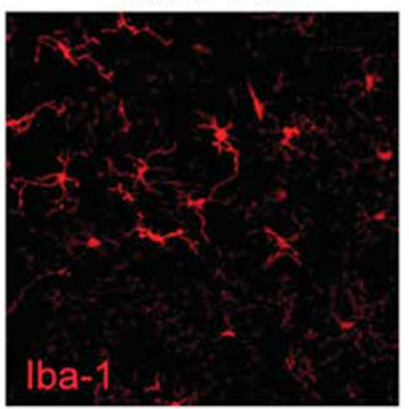

b

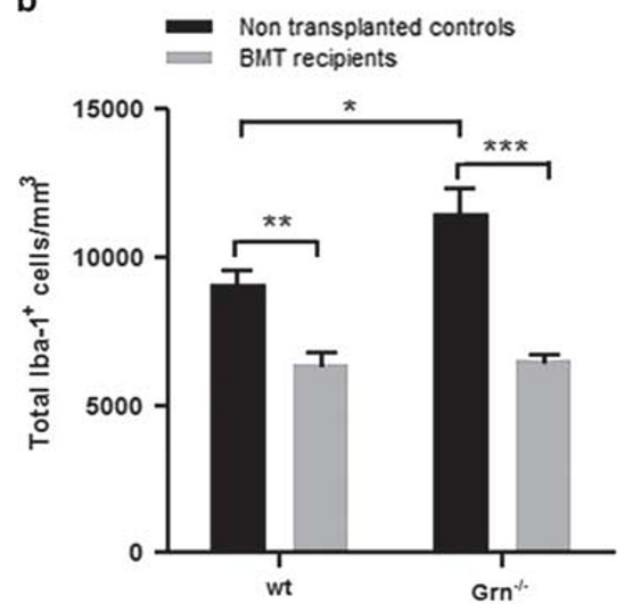

Figure 5 Microgliosis in $\mathrm{Grn}^{-1-}$ mice was erased by BMT. (a) Representative images of Iba-1 immunofluorescence in cerebral cortex of chimeric mice and their non-transplanted controls. Scale bars: $50 \mu \mathrm{m}$. (b) Unbiased stereological quantitation of microglia (total lba- $1^{+}$ microglia/ $/ \mathrm{mm}^{3}$ ) in the cerebral cortex reveals a significant increase in Iba- ${ }^{+}$cells in $\mathrm{Grn}^{-1-}$ compared with wt mice $\left.{ }^{*} P<0.05\right)$. BMT had a significant effect on microglia density $\left({ }^{* *} P<0.001\right)$ with Bonferronicorrected posttests showing significantly reduced microglia density both in wt-GFP $\rightarrow \mathrm{Grn}^{-1-}$ mice vs Grn mice $\left({ }^{* *} \mathrm{P}<0.001\right)$ and in wt-GFP $\rightarrow$ wt mice vs wt mice ( $* * P<0.01)$. Error bars show the means \pm s.e.m., $n=4-6$. Two-way ANOVA was performed using the Bonferroni post hoc test.

cortex of $\mathrm{Grn}^{-1-}$ mice. Our results showed that wt BMT partially reconstituted progranulin in the periphery and in cerebral cortex of $\mathrm{Grn}^{-1-}$ mice, and that this was sufficient to reverse, either in part or in full, ex vivo and in vivo measures of exaggerated immune response that are characteristic of $\mathrm{Grn}^{-1-}$ mouse cerebral cortex.
Similar to previous results from others and us, myeloablative BMT led to near complete peripheral (blood) engraftment and partial engraftment in the cerebral cortex with microglia. ${ }^{62,67-69}$ New to these experiments, we observed by both flow cytometry and stereologic cell counting that cerebral cortical engraftment in wt-GFP $\rightarrow \mathrm{Grn}^{-1-}$ mice was about one-half of wt-GFP $\rightarrow$ wt mice. We investigated four factors that might contribute to reduced engraftment in wt-GFP $\rightarrow \mathrm{Grn}^{-1-}$ mice, and observed that only cerebral cortical CXCL10 levels were selectively increased in wt$\mathrm{GFP} \rightarrow$ wt but not in wt-GFP $\rightarrow \mathrm{Gr}^{-1-}$ mice. Myeloablative conditioning increases CXCL10 expression in brain where its levels correlate with myeloid cell recruitment. ${ }^{70}$ CXCR3/ CXCL10 signaling has been suggested to be one of the most relevant pathways in the attraction of immune cells such as monocytes/macrophages, $\mathrm{T}$ cells, natural killer (NK) cells, and dendritic cells to inflamed tissues. ${ }^{71}$ It also has been demonstrated that CXCR3/CXCL10 signaling is crucial in microglia recruitment. ${ }^{72}$ Although not exhaustive, these results provide one clue about a potentially important molecular mechanism to enhance brain engraftment following BMT.

Since peripheral engraftment was near complete, it was not surprising that plasma progranulin concentration in wt-GFP $\rightarrow$ wt recipients was similar to non-transplanted wt mice. However, plasma progranulin concentration in $\mathrm{Grn}^{-1-}$ recipients was only about $23 \%$ of wt mice, indicating that cellular sources other than BMT-derived cells contribute the majority of progranulin in mouse plasma. Results were similar in cerebral cortex where only about $8 \%$ of wt progranulin concentration was detected in wt-GFP $\rightarrow \mathrm{Grn}^{-1-}$ mice. A likely additional source of progranulin here is neurons. Despite this relatively low level of cerebral cortical progranulin, we demonstrated biological activity in both ex vivo and in vivo assays of enhanced immune response in $\mathrm{Grn}^{-1-}$ mice. Indeed, our ex vivo assay of TLR4 activation of cerebral slices showed $\sim 40 \%$ rescue in $\mathrm{wt}-\mathrm{GFP} \rightarrow \mathrm{Grn}^{-1-}$ mice from the progranulin deficiencyinduced exaggerated immune response. It is worth noting that there was a trend of increased immune response in BMT wt recipients, perhaps as a result of radiation, and thus this reduction occurred even in the face of a potentially larger response. In addition, our three in vivo assays showed complete reversal of the effects of progranulin deficiency in wt$\mathrm{GFP} \rightarrow \mathrm{Grn}^{-1-}$ mice with respect to total cerebral cortical microglia CD126 or CD36 expression, and cerebral cortical microgliosis.

Although not a focus of our study, our results do add to our knowledge of the immune modulatory effects of progranulin deficiency in cerebral cortex. Grn ${ }^{-1-}$ mice had increased microglial CD126 and CD36 expression that was completely reversed by BMT, but not significantly increased MHC class II. Indeed, others have reported that deletion of progranulin increases macrophage CD36 expression and induces local inflammation. ${ }^{73}$ Several studies have linked 
progranulin activity with IL-6 expression, ${ }^{74,75}$ however, we are unaware of previous work linking progranulin deficiency and increased expression of CD126, a receptor of IL-6. As expected from previous work, wt microglia MHC class II expression was increased following $\mathrm{BMT},{ }^{48}$ and this effect was equivalent in wt and $\mathrm{Grn}^{-1-}$ BMT recipients. Finally, we investigated differences in donor $v s$ recipient microglia with respect to MHC class II, CD126, and CD36 expression. Our results confirm the work of others and us by showing that donor and recipient microglia are not identical, ${ }^{48,76}$ and suggest a complex interplay between source of mononuclear cell and environment of target tissue with respect to microglial expression of these three molecules. Other factors such as differences between wt and $\mathrm{Grn}^{-1-}$ mice in homing efficacy of extraneous cells to the CNS might also contribute to these observed differences.

We recognize that our experiments are proof-of-concept, and demonstrate only proximate biochemical effects of wt BMT in cerebral cortex of $\mathrm{Grn}^{-1-}$ mice, but not therapeutic benefit. We think that this is an appropriate first step because, even though $\mathrm{Grn}^{-1-}$ mice are by far the dominant experimental model in FTLD-TDP research, ${ }^{77}$ it is not at all clear that the behavioral changes in $\mathrm{Gr}^{-1-}$ mice, which would be the end points for therapeutic studies, are reflective of pathologic processes in patients with FTLD-TDP from GRN mutations. Indeed, it will be more appropriate to conduct future behavioral studies in $\mathrm{Grn}^{+/-}$mice following BMT. Other limitations of our study are that we did not demonstrate durability of the partial reconstitution of progranulin in cerebral cortex following BMT; however, we have previously shown that the proportion of microglia in cerebral cortex that are BM-derived increases over time up to 8 months post BMT (our latest time point examined). ${ }^{67}$ Finally, we focused on immune response in the cerebral cortex as a measure of bioactivity of BMT because engrafted cells are microglia; however, we appreciate that these are surrogates and that specific action(s) of progranulin and its cleavage products that are relevant to FTLD may or may not involve immune regulation.

We envision BMT as a stem cell-based approach to prevent or to treat neurodegenerative diseases related to microglial dysfunction, like TREM2 mutations in some forms of $\mathrm{AD}$, or molecular deficiencies that may be partially reversed by microglia, like GRN haploinsufficiency in some forms of FTD. Myeloablative BMT carries significant morbidity and mortality that should not be undertaken except to treat lifethreatening illnesses. Neuronal ceroid lipofuscinosis-11 and perhaps FTD from GRN mutations might be appropriate indications. Recent studies have reported success with BMT in a murine model of infantile neuronal ceroid lipofuscinosis ${ }^{78}$ however, older studies with animal models or volunteers who had different forms of neuronal ceroid lipofuscinosis were at best modestly successful. ${ }^{79,80}$ We were unaware of any BMT approach to alleviating neuronal ceroid lipofuscinosis-11. Non-myeloablative BMT carries substantially less morbidity and mortality, and has been attempted in the treatment of several non-cancerous diseases that do not require ablation of the marrow. We have shown in mice that, although non-myeloablative BMT engrafts the periphery with efficiency of about $90 \%$ at 8 months post transplant, it does not engraft brain. ${ }^{81}$ There are no data on the interactions between peripheral and central progranulin, so we cannot predict the likelihood that non-myeloablative BMT might ameliorate biochemical deficits in cerebral cortex of $\mathrm{Grn}^{-1-}$ mice or behavioral changes in $\mathrm{Grn}^{+/-}$mice; these complicated experiments are underway.

In summary, our results confirm a pro-inflammatory effect in vivo and in ex vivo preparations of cerebral cortex of $\mathrm{Grn}^{-1-}$ mice that can be partially to fully reversed at 5 months post transplant following wt BMT at 2 months of age. Although several important issues remain to be investigated, these results raise the possibility of BMT as a potential intervention for inherited degenerative diseases caused by progranulin deficiency.

\section{ACKNOWLEDGMENTS}

This study was supported by NIH grants P50AG05136 and T32AG000258 (EC), and by the Nancy and Buster Alvord Endowment. We would like to thank Ms Aimee Schantz and Ms Carol Arnold for administrative support and Dr Martin Darvas for helpful discussions.

\section{DISCLOSURE/CONFLICT OF INTEREST}

The authors declare no conflict of interest.

1. Smith KR, Damiano J, Franceschetti $\mathrm{S}$, et al. Strikingly different clinicopathological phenotypes determined by progranulin-mutation dosage. Am J Hum Genet 2012;90:1102-1107.

2. Ahmed Z, Sheng $\mathrm{H}, \mathrm{Xu}$ YF, et al. Accelerated lipofuscinosis and ubiquitination in granulin knockout mice suggest a role for progranulin in successful aging. Am J Pathol 2010;177:311-324.

3. Yu CE, Bird TD, Bekris LM, et al. The spectrum of mutations in progranulin: a collaborative study screening 545 cases of neurodegeneration. Arch Neurol 2010;67:161-170.

4. Gass J, Cannon A, Mackenzie IR, et al. Mutations in progranulin are a major cause of ubiquitin-positive frontotemporal lobar degeneration. Hum Mol Genet 2006;15:2988-3001.

5. Mackenzie IR. The neuropathology and clinical phenotype of FTD with progranulin mutations. Acta Neuropathol 2007;114:49-54.

6. Baker M, Mackenzie IR, Pickering-Brown SM, et al. Mutations in progranulin cause tau-negative frontotemporal dementia linked to chromosome 17. Nature 2006;442:916-919.

7. Cruts M, Gijselinck I, van der Zee J, et al. Null mutations in progranulin cause ubiquitin-positive frontotemporal dementia linked to chromosome 17q21. Nature 2006;442:920-924.

8. Arai T, Hasegawa M, Akiyama $\mathrm{H}$, et al. TDP-43 is a component of ubiquitin-positive tau-negative inclusions in frontotemporal lobar degeneration and amyotrophic lateral sclerosis. Biochem Biophys Res Commun 2006;351:602-611.

9. Neumann M, Sampathu DM, Kwong LK, et al. Ubiquitinated TDP-43 in frontotemporal lobar degeneration and amyotrophic lateral sclerosis. Science 2006;314:130-133.

10. Van Deerlin VM, Sleiman PM, Martinez-Lage M, et al. Common variants at 7 p21 are associated with frontotemporal lobar degeneration with TDP-43 inclusions. Nat Genet 2010;42:234-239.

11. Cairns NJ, Neumann M, Bigio EH, et al. TDP-43 in familial and sporadic frontotemporal lobar degeneration with ubiquitin inclusions. Am J Pathol 2007;171:227-240.

12. Gotzl JK, Mori K, Damme M, et al. Common pathobiochemical hallmarks of progranulin-associated frontotemporal lobar degeneration 
and neuronal ceroid lipofuscinosis. Acta Neuropathol 2014;127: 845-860.

13. Ahmed $\mathrm{Z}$, Mackenzie $I R$, Hutton $M L$, et al. Progranulin in frontotemporal lobar degeneration and neuroinflammation. J Neuroinflammation 2007;4:7.

14. Seeley WW. Selective functional, regional, and neuronal vulnerability in frontotemporal dementia. Curr Opin Neurol 2008;21:701-707.

15. Daniel R, Daniels E, He Z, et al. Progranulin (acrogranin/PC cell-derived growth factor/granulin-epithelin precursor) is expressed in the placenta, epidermis, microvasculature, and brain during murine development. Dev Dyn 2003;227:593-599.

16. Matsuwaki T, Asakura R, Suzuki $M$, et al. Age-dependent changes in progranulin expression in the mouse brain. J Reprod Dev 2011;57: 113-119.

17. Petkau TL, Neal SJ, Orban PC, et al. Progranulin expression in the developing and adult murine brain. J Comp Neurol 2010;518:39313947.

18. Nicholson AM, Finch NA, Thomas CS, et al. Progranulin protein levels are differently regulated in plasma and CSF. Neurology 2014;82: 1871-1878.

19. Finch N, Baker $M$, Crook R, et al. Plasma progranulin levels predict progranulin mutation status in frontotemporal dementia patients and asymptomatic family members. Brain 2009;132:583-591.

20. Philips T, De Muynck L, Thu HN, et al. Microglial upregulation of progranulin as a marker of motor neuron degeneration. J Neuropathol Exp Neurol 2010;69:1191-1200.

21. He Z, Ong $\mathrm{CH}$, Halper J, et al. Progranulin is a mediator of the wound response. Nat Med 2003;9:225-229.

22. Yin F, Banerjee $R$, Thomas B, et al. Exaggerated inflammation, impaired host defense, and neuropathology in progranulin-deficient mice. J Exp Med 2010;207:117-128.

23. Kao AW, Eisenhut RJ, Martens LH, et al. A neurodegenerative disease mutation that accelerates the clearance of apoptotic cells. Proc Natl Acad Sci USA 2011;108:4441-4446.

24. Gass J, Lee WC, Cook C, et al. Progranulin regulates neuronal outgrowth independent of sortilin. Mol Neurodegener 2012;7:33.

25. Van Damme P, Van Hoecke A, Lambrechts D, et al. Progranulin functions as a neurotrophic factor to regulate neurite outgrowth and enhance neuronal survival. J Cell Biol 2008;181:37-41.

26. Ryan CL, Baranowski DC, Chitramuthu BP, et al. Progranulin is expressed within motor neurons and promotes neuronal cell survival. BMC Neurosci 2009:10:130.

27. Tapia L, Milnerwood A, Guo A, et al. Progranulin deficiency decreases gross neural connectivity but enhances transmission at individual synapses. J Neurosci 2011;31:11126-11132.

28. $\mathrm{Hu} F$, Padukkavidana $\mathrm{T}$, Vaegter $\mathrm{CB}$, et al. Sortilin-mediated endocytosis determines levels of the frontotemporal dementia protein, progranulin. Neuron 2010;68:654-667.

29. Zhu J, Nathan C, Jin W, et al. Conversion of proepithelin to epithelins: roles of SLPI and elastase in host defense and wound repair. Cell 2002;111:867-878.

30. Suh HS, Choi N, Tarassishin L, et al. Regulation of progranulin expression in human microglia and proteolysis of progranulin by matrix metalloproteinase-12 (MMP-12). PLoS ONE 2012;7:e35115.

31. Kessenbrock K, Frohlich L, Sixt $M$, et al. Proteinase 3 and neutrophil elastase enhance inflammation in mice by inactivating antiinflammatory progranulin. J Clin Invest 2008;118:2438-2447.

32. Wang $X, L i X, X u L$, et al. Up-regulation of secretory leukocyte protease inhibitor (SLPI) in the brain after ischemic stroke: adenoviral expression of SLPI protects brain from ischemic injury. Mol Pharmacol 2003;64:833-840.

33. He Z, Ismail A, Kriazhev L, et al. Progranulin (PC-cell-derived growth factor/acrogranin) regulates invasion and cell survival. Cancer Res 2002;62:5590-5596.

34. Okura H, Yamashita S, Ohama T, et al. HDL/apolipoprotein A-I binds to macrophage-derived progranulin and suppresses its conversion into proinflammatory granulins. J Atheroscler Thromb 2010;17:568-577.

35. Tang $W, L u Y$, Tian QY, et al. The growth factor progranulin binds to TNF receptors and is therapeutic against inflammatory arthritis in mice. Science 2011:332:478-484.

36. Kayasuga $Y$, Chiba $S$, Suzuki $M$, et al. Alteration of behavioural phenotype in mice by targeted disruption of the progranulin gene. Behav Brain Res 2007;185:110-118.
37. Yin $\mathrm{F}$, Dumont $\mathrm{M}$, Banerjee $\mathrm{R}$, et al. Behavioral deficits and progressive neuropathology in progranulin-deficient mice: a mouse model of frontotemporal dementia. Faseb J 2010;24:4639-4647.

38. Ghoshal N, Dearborn JT, Wozniak DF, et al. Core features of frontotemporal dementia recapitulated in progranulin knockout mice. Neurobiol Dis 2012;45:395-408.

39. Filiano AJ, Martens LH, Young AH, et al. Dissociation of frontotemporal dementia-related deficits and neuroinflammation in progranulin haploinsufficient mice. J Neurosci 2013;33:5352-5361.

40. Lee WC, Almeida S, Prudencio $M$, et al. Targeted manipulation of the sortilin-progranulin axis rescues progranulin haploinsufficiency. Hum Mol Genet 2014:23:1467-1478.

41. Duran-Struuck R, Dysko RC. Principles of bone marrow transplantation (BMT): providing optimal veterinary and husbandry care to irradiated mice in BMT studies. J Am Assoc Lab Anim Sci 2009;48:11-22.

42. Li XH, Ghosh SP, Ha CT, et al. Delta-tocotrienol protects mice from radiation-induced gastrointestinal injury. Radiat Res 2013;180: 649-657.

43. Mouthon MA, Van der Meeren A, Gaugler MH, et al. Thrombopoietin promotes hematopoietic recovery and survival after high-dose whole body irradiation. Int J Radiat Oncol Biol Phys 1999;43:867-875.

44. Cardona $A E$, Huang D, Sasse ME, et al. Isolation of murine microglial cells for RNA analysis or flow cytometry. Nat Protoc 2006;1:1947-1951.

45. Ford AL, Goodsall AL, Hickey WF, et al. Normal adult ramified microglia separated from other central nervous system macrophages by flow cytometric sorting. Phenotypic differences defined and direct ex vivo antigen presentation to myelin basic protein-reactive CD4 $+\mathrm{T}$ cells compared. J Immunol 1995;154:4309-4321.

46. Havenith CE, Askew D, Walker WS. Mouse resident microglia: isolation and characterization of immunoregulatory properties with naive CD4 + and CD8 + T-cells. Glia 1998:22:348-359.

47. Overton WR. Modified histogram subtraction technique for analysis of flow cytometry data. Cytometry 1988:9:619-626.

48. Yang Y, Cudaback E, Jorstad NL, et al. APOE3, but not APOE4, bone marrow transplantation mitigates behavioral and pathological changes in a mouse model of Alzheimer disease. Am J Pathol 2013;183:905-917.

49. Yang Y, Shiao C, Hemingway JF, et al. Suppressed retinal degeneration in aged wild type and APPswe/PS1DeltaE9 mice by bone marrow transplantation. PLoS ONE 2013;8:e64246.

50. Simard AR, Rivest S. Bone marrow stem cells have the ability to populate the entire central nervous system into fully differentiated parenchymal microglia. Faseb J 2004;18:998-1000.

51. Derecki NC, Cronk JC, Lu Z, et al. Wild-type microglia arrest pathology in a mouse model of Rett syndrome. Nature 2012;484:105-109.

52. Priller J, Flugel $\mathrm{A}$, Wehner $\mathrm{T}$, et al. Targeting gene-modified hematopoietic cells to the central nervous system: use of green fluorescent protein uncovers microglial engraftment. Nat Med 2001;7:1356-1361.

53. Li X, Cudaback E, Keene CD, et al. Suppressed microglial E prostanoid receptor 1 signaling selectively reduces tumor necrosis factor alpha and interleukin 6 secretion from toll-like receptor 3 activation. Glia 2011:59:569-576.

54. Cudaback E, Li X, Yang Y, et al. Apolipoprotein C-I is an APOE genotype-dependent suppressor of glial activation. J Neuroinflammation 2012;9:192.

55. Dantzer R, O'Connor JC, Freund GG, et al. From inflammation to sickness and depression: when the immune system subjugates the brain. Nat Rev Neurosci 2008:9:46-56.

56. Fernando MR, Reyes JL, lannuzzi J, et al. The pro-inflammatory cytokine, interleukin-6, enhances the polarization of alternatively activated macrophages. PLoS ONE 2014;9:e94188.

57. Shie FS, Neely MD, Maezawa I, et al. Oxidized low-density lipoprotein is present in astrocytes surrounding cerebral infarcts and stimulates astrocyte interleukin-6 secretion. Am J Pathol 2004;164:1173-1181.

58. Oberg HH, Wesch $\mathrm{D}$, Grussel $\mathrm{S}$, et al. Differential expression of CD126 and CD130 mediates different STAT-3 phosphorylation in CD4 + CD25- and CD25high regulatory T cells. Int Immunol 2006;18: 555-563.

59. Coraci IS, Husemann J, Berman JW, et al. CD36, a class B scavenger receptor, is expressed on microglia in Alzheimer's disease brains and can mediate production of reactive oxygen species in response to beta-amyloid fibrils. Am J Pathol 2002;160:101-112. 
60. Erdman LK, Cosio G, Helmers AJ, et al. CD36 and TLR interactions in inflammation and phagocytosis: implications for malaria. J Immunol 2009;183:6452-6459.

61. El Khoury JB, Moore KJ, Means TK, et al. CD36 mediates the innate host response to beta-amyloid. J Exp Med 2003;197:1657-1666.

62. Djukic M, Mildner A, Schmidt $H$, et al. Circulating monocytes engraft in the brain, differentiate into microglia and contribute to the pathology following meningitis in mice. Brain 2006;129:2394-2403.

63. Ward ME, Miller BL. Potential mechanisms of progranulin-deficient FTLD. J Mol Neurosci 2011;45:574-582.

64. Martens LH, Zhang J, Barmada SJ, et al. Progranulin deficiency promotes neuroinflammation and neuron loss following toxininduced injury. J Clin Invest 2012;122:3955-3959.

65. Alberici A, Archetti S, Pilotto A, et al. Results from a pilot study on amiodarone administration in monogenic frontotemporal dementia with granulin mutation. Neurol Sci 2014;35:1215-1219.

66. Van Kampen JM, Baranowski D, Kay DG. Progranulin gene delivery protects dopaminergic neurons in a mouse model of Parkinson's disease. PLoS ONE 2014;9:e97032.

67. Keene CD, Chang RC, Lopez-Yglesias AH, et al. Suppressed accumulation of cerebral amyloid \{beta\} peptides in aged transgenic Alzheimer's disease mice by transplantation with wild-type or prostaglandin E2 receptor subtype 2-null bone marrow. Am J Pathol 2010;177:346-354.

68. Lampron A, Lessard M, Rivest S. Effects of myeloablation, peripheral chimerism, and whole-body irradiation on the entry of bone marrowderived cells into the brain. Cell Transplant 2012;21:1149-1159.

69. Malm TM, Koistinaho M, Parepalo $M$, et al. Bone-marrow-derived cells contribute to the recruitment of microglial cells in response to betaamyloid deposition in APP/PS1 double transgenic Alzheimer mice. Neurobiol Dis 2005;18:134-142.

70. Mildner A, Schmidt $H$, Nitsche $M$, et al. Microglia in the adult brain arise from Ly-6ChiCCR2 + monocytes only under defined host conditions. Nat Neurosci 2007;10:1544-1553.
71. Bondar C, Araya RE, Guzman L, et al. Role of CXCR3/CXCL10 axis in immune cell recruitment into the small intestine in celiac disease. PLoS ONE 2014;9:e89068.

72. Rappert A, Bechmann I, Pivneva T, et al. CXCR3-dependent microglial recruitment is essential for dendrite loss after brain lesion. J Neurosci 2004;24:8500-8509.

73. Kawase R, Ohama $T$, Matsuyama A, et al. Deletion of progranulin exacerbates atherosclerosis in ApoE knockout mice. Cardiovasc Res 2013;100:125-133.

74. Matsubara T, Mita A, Minami K, et al. PGRN is a key adipokine mediating high fat diet-induced insulin resistance and obesity through IL-6 in adipose tissue. Cell Metab 2012;15:38-50.

75. Jian J, Konopka J, Liu C. Insights into the role of progranulin in immunity, infection, and inflammation. J Leukoc Biol 2013;93: 199-208.

76. Mildner A, Schlevogt B, Kierdorf $\mathrm{K}$, et al. Distinct and non-redundant roles of microglia and myeloid subsets in mouse models of Alzheimer's disease. J Neurosci 2011;31:11159-11171.

77. Roberson ED. Mouse models of frontotemporal dementia. Ann Neurol 2012;72:837-849.

78. Macauley SL, Roberts MS, Wong AM, et al. Synergistic effects of central nervous system-directed gene therapy and bone marrow transplantation in the murine model of infantile neuronal ceroid lipofuscinosis. Ann Neurol 2012;71:797-804.

79. Lake BD, Steward CG, Oakhill A, et al. Bone marrow transplantation in late infantile Batten disease and juvenile Batten disease. Neuropediatrics 1997;28:80-81.

80. Deeg HJ, Shulman HM, Albrechtsen D, et al. Batten's disease: failure of allogeneic bone marrow transplantation to arrest disease progression in a canine model. Clin Genet 1990;37:264-270.

81. Yang Y, Jorstad NL, Shiao C, et al. Perivascular, but not parenchymal, cerebral engraftment of donor cells after non-myeloablative bone marrow transplantation. Exp Mol Pathol 2013;95:7-17. 